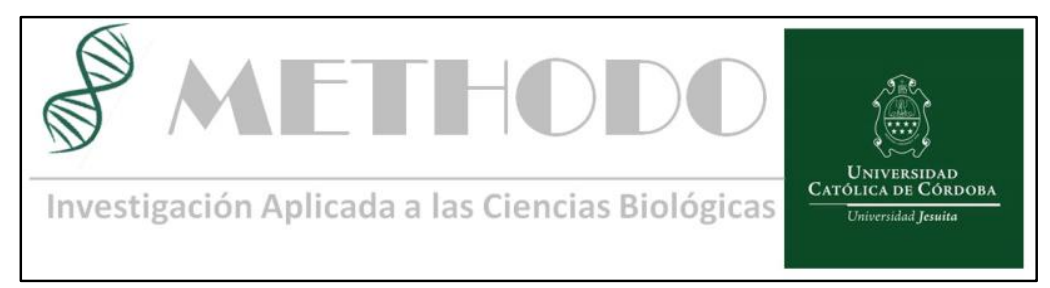

CASO CLINICO Methodo 2018;3(3): 88-92

DOI: 10.22529/me.2018.3(3)08

\title{
Técnica innovadora semidirecta de Individualización con endoposte de fibra
}

\section{Innovative chairside technique of Individualized fiber post}

\author{
Daniel Torassa. Prof Dr. ${ }^{1}$ \\ ${ }^{1}$ Profesor Titular Cátedra Clínica de Prótesis I. Carrera de Odontología. Facultad de Ciencias de la Salud. Universidad Católica de \\ Córdoba. No existe financiación, conflicto de intereses, ni patrocinante en la realización de este caso clínico.
}

\section{Resumen:}

La restauración con sistema de endoposte de fibra mediante técnica de individualización o anatómica es conocida desde hace tiempo.

Se presenta una alternativa para ser realizada de manera semidirecta mediante la confección de un modelo flexible translúcido que permite reducir algunos inconvenientes de la técnica directa de individualización.

El material empleado para el modelo es un material de bajo costo y accesible para cualquier profesional que desee emplear esta alternativa de restauración.

\begin{abstract}
:
Anatomical or individualized fiber post technique has been used for many years now.

An alternative is presented to be done chairside with a flexible and translucid model obtained that allows to reduce some drawbacks of the direct anatomical technique.

The material used is less expensive than other options and might be used by any professional that want to learn this technique.
\end{abstract}

\section{Introducción:}

La restauración de elementos dentarios con sistemas de endopostes no metálicos ha evolucionado en los últimos años permitiendo ampliar las posibilidades de reconstrucción con diferentes técnicas y materiales.

Los sistemas de endopostes no metálicos, en particular de fibra de vidrio y fibra de cuarzo son los de uso más frecuente en la actualidad por sus propiedades físicas y biomecánicas.

Estos endopostes pueden ser utilizados para rehabilitar las situaciones clínicas que enfrentamos en elementos dentarios con tratamiento de endodoncia y que requieren de un sistema de poste para sostener y conectar el núcleo del pilar coronario con una restauración coronaria total. ${ }^{1-2}$ En elementos dentarios con escaso remanente muchos clínicos prefieren seguir utilizando postes o pernos metálicos por la resistencia del perno y la dificultad de lograr adhesión a una estructura dentaria reducida. ${ }^{3}$

Para poder solucionar con un sistema de endoposte no metálico estas instancias clínicas desfavorables se han propuesto técnicas como la de Poste individualizado o también denominada técnica de poste anatómico. ${ }^{4-5}$

El empleo de materiales flexibles para confeccionar un modelo y efectuar el procedimiento indirecto para luego ser cementado es conocido y empleado desde hace tiempo en otras técnicas restauradoras como son las incrustaciones o los provisionales. ${ }^{6}$

Es objetivo de este caso clínico mostrar una alternativa de técnica semidirecta con un protocolo de trabajo que utiliza un modelo confeccionado con un material flexible y de bajo costo que permite polimerizar a través del mismo dada su naturaleza translúcida y reduce los tiempos de ejecución favoreciendo la aplicación semidirecta en el consultorio. ${ }^{7}$ 


\section{Caso Clínico}

Se presenta a consulta un paciente de sexo masculino con el desprendimiento de una restauración coronaria y perno metálico colado en posición elemento 22 con remanente coronario reducido y conducto preparado con espesor dentinario radicular reducido. (Figura 1)

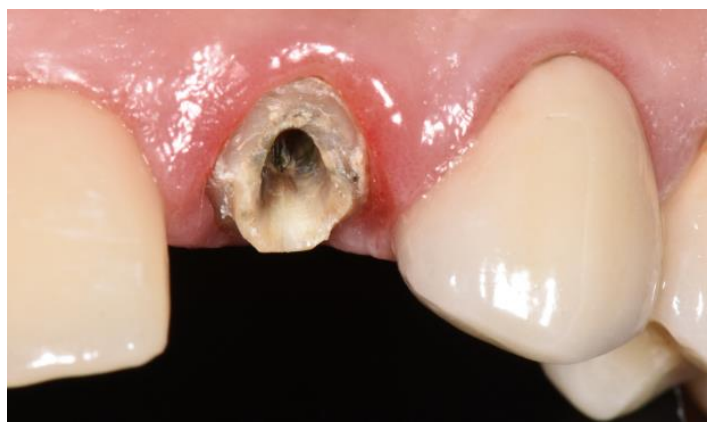

Figura 1. Estructura dentaria remanente y espacio del conducto preparado.

En el diagnóstico clínico general, donde es necesario valorar aspectos específicos del elemento dentario como la condición del tratamiento de endodoncia, periodontal, remanente dentario, también analizamos el posible componente biomecánico que deberá soportar, situación oclusal, historia odontológica en toda la cavidad bucal, todos elementos que nos permiten generar planes de tratamiento adecuados a la situación clínica a resolver.

El remanente coronario presente, aunque reducido, permitía generar un efecto de encapsulamiento o ferrule que es fundamental para la predictibilidad de la futura restauración.

El espacio del conducto se presenta con un diámetro ampliado y con una estructura dentinaria del tejido contaminada con corrosión y tratamiento de endodoncia de larga data, aspectos importantes a considerar al momento de decidir el protocolo de cementado del sistema de poste a utilizar.

Para la rehabilitación se decide emplear técnica de restauración con sistema de poste de fibra de cuarzo y núcleo de resina con técnica semidirecta realizada en el momento a partir de la reproducción del terreno protésico y obtención de modelo flexible para la confección del poste y núcleo coronario sobre modelo.

Se efectúa la preparación del conducto con el sistema de fresas del diámetro de poste seleccionado en este caso clínico un poste tamaño numero 2 (RTD Macrolock St Egreve, Francia.) dejando un sellado apical de $3 \mathrm{~mm}$ como mínimo para optimizar la longitud de retención del poste empleado.

La reproducción del terreno se realizó mediante técnica simultánea con silicona por adición (President
, Coltène/Whaledent AG, Suiza) consistencia pesada y liviana, utilizando el propio endoposte como alma de soporte para la silicona liviana que reproduce el espacio interno del conducto para evitar posibles distorsiones al momento de la confección del modelo. (Figura 2)

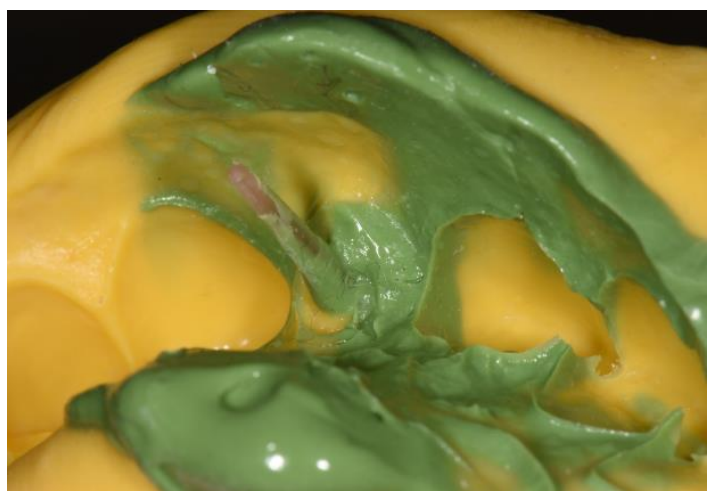

Figura 2. Impresión del conducto y remanente dentario con endoposte como soporte del material de impresión.

Obtenida la impresión se realiza el vaciado con material termoplástico (HMA por sus siglas en inglés: Hot Melt Material) que se provee en varillas para utilizar con una pistola dispensadora que calienta el material (Suprabond Hot Melt) y lo convierte en estado fluido en consistencia de gel que luego endurece nuevamente a temperatura ambiente y por su naturaleza química consistente en co-polímeros de acetato de vinyl etileno y aditivos no se une a la silicona por adición. (Figura 3)

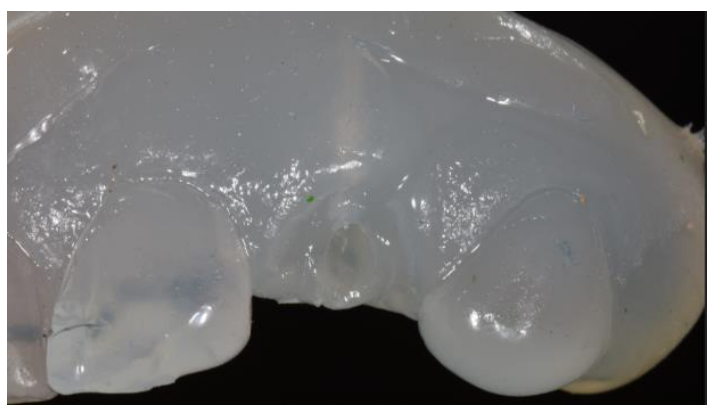

Figura 3. Modelo Flexible translucido.

Realizado el modelo flexible y translúcido, se retira el endoposte de la impresión donde se lo utilizó como soporte de la silicona que registro el espacio del conducto.

Se efectúa la preparación de la superficie del poste mediante desengrasado con alcohol durante 30 segundos, se lava con spray de agua y aire libre de aceite, secado durante 10 segundos.

Se aplica resina de enlace o bonding sobre la superficie del poste, se elimina exceso con spray de aire desde el sector apical hacia coronario y se polimeriza durante 40 segundos por todas sus caras. 
Posteriormente se aplica resina fotopolimerizable sobre el poste para copiar la superficie interna del conducto donde la adaptación del poste dada su conformación circular deja un espacio.

Se polimeriza por 40 segundos desde coronario y a través del modelo que por su translucidez permite una polimerización limitada, pero que logra el endurecimiento del material en una primera instancia para ser retirado y polimerizado de manera adecuada fuera del modelo.

Se continúa aplicando resina fotopolimerizable en la porción coronaria para conformar el pilar coronario de soporte. En cada incremento se polimeriza por 40 segundos en todas las caras expuestas.

Una vez conformado el núcleo coronario se retira del modelo flexible (Figura 4) y se procede a la prueba en boca sobre el pilar dentario preparado.

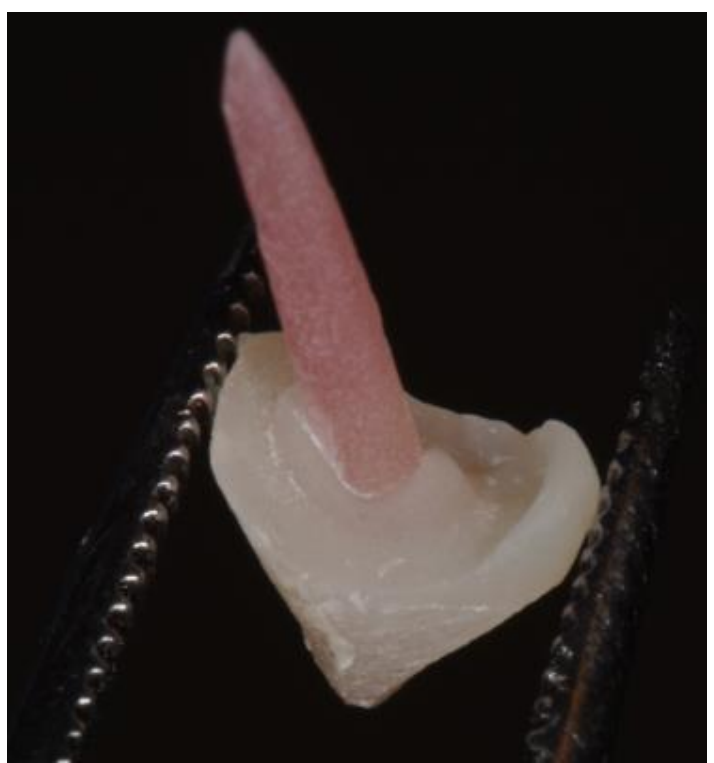

Figura 4. Poste y reconstrucción con resina del pilar coronario.

Una vez corroborado el ajuste, se efectúa el cementado. (Figura 5)

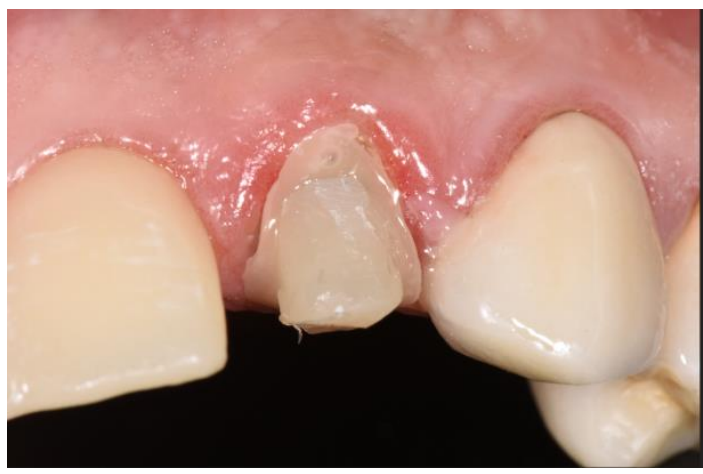

Figura 5. Poste y núcleo cementado con cemento autoadhesivo.

En esta situación clínica por la capacidad de ajuste y estabilidad del poste logrado por la individualización permite el cementado con diferentes opciones siendo la seleccionada en esta instancia el protocolo con cemento autoadhesivo. (Solocem- Coltène/Whaledent AG, Suiza).

Se polimeriza desde cervical y coronario para lograr la acción de fotopolimerización del medio de cementado en las áreas donde sea posible esta acción. ${ }^{8}$

Se realiza la preparación del pilar y remanente dentario para una restauración de cerámica sin metal, provisionalización con resina bis-acrílica (Cooltemp-Coltène/Whaledent AG, Suiza), a partir de matriz previamente confeccionada y se efectúa impresión definitiva con técnica de doble impresión. (Figuras 6-7)

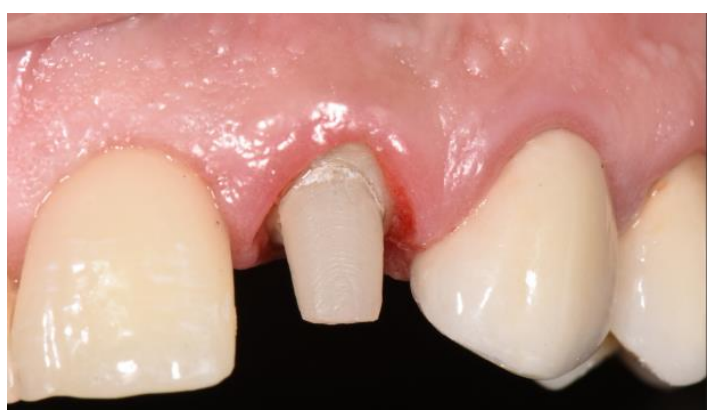

Figura 6. Pilar y remanente dentario preparado.

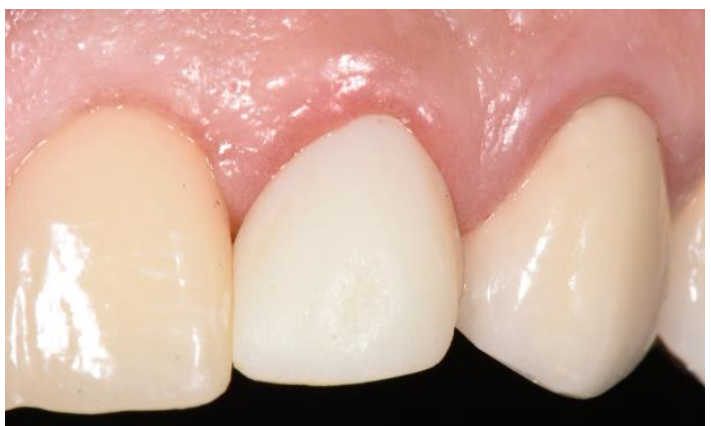

Figura 7. Provisionalización inmediata con resina Bis Acrílica.

Confeccionada la restauración coronaria cerámica en laboratorio se realizó el cementado de la misma con técnica de cementado adhesivo mediante cemento de resina dual. (Figura 8)

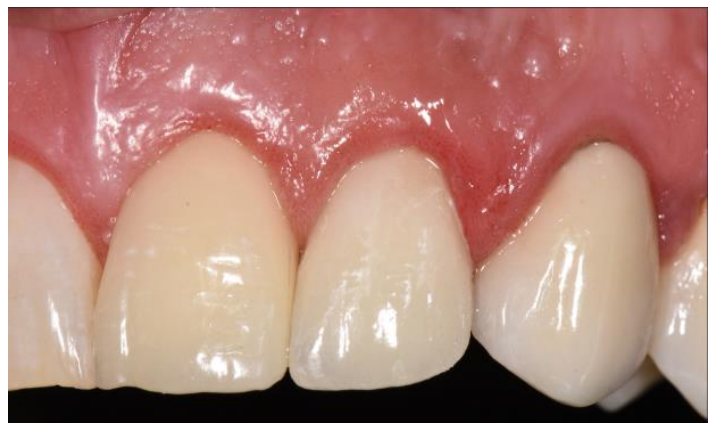

Figura 8. Restauración definitiva cerámica.

Revista Methodo: Investigación Aplicada a las Ciencias Biológicas. Universidad Católica de Córdoba Jacinto Ríos 571 Bo Gral. Paz. X5004FXS. Córdoba. Argentina. Tel.: (54) 3514517299 / Correo: methodo@ucc.edu.ar / Web: methodo.ucc.edu.ar | CASO CLINICO Methodo 2018;3(3): 88-92 


\section{Discusión:}

La restauración de elementos que requieren de un sistema de poste para soporte de restauración coronaria total ha evolucionado permitiendo el empleo de soluciones biomecánicas y biológicas compatibles con los tejidos dentarios, aun en situaciones con escaso remanente.

Por años hemos limitado las indicaciones clínicas de los sistemas de poste no metálicos a elementos dentarios con un remanente que fuera favorable, siendo este término poco preciso y abierto a interpretaciones clínicas diversas.

Por otro lado, contraindicamos su utilización en casos donde el remanente dentario coronario es escaso y con la misma ausencia de aclaración sobre las condiciones o cantidades de lo que el término implica.

En ninguno de los dos casos, favorable remanente o escaso remanente la literatura muestra publicaciones que justifiquen dicha indicación o al menos los resultados son contradictorios en muchos casos.

En las posibilidades de tratamiento de este caso clínico presentado surgen distintas opciones que podrían ser utilizadas, desde la posible exodoncia y resolución con implante o la alternativa de un poste colado convencional hasta la restauración con un sistema de poste biomecánicamente compatible con los tejidos como el utilizado.

La valoración diagnóstica de cualquier situación clínica, aún el caso de restauración dentaria unitaria requiere de analizar todos aspectos funcionales y biomecánicos.

Esto significa incorporar en el diagnóstico las características del sector a rehabilitar, si es anterior o posterior, presencia de facetas patológicas de desgaste, historia de rehabilitación que identifiquemos al momento de observar la situación clínica puedan ser elementos que nos ayuden a definir el tratamiento a establecer como el apropiado para la situación clínica específica a tratar.

La técnica específica descripta es una opción a otras posibilidades de restauración con poste de fibra realizadas directas en boca como la técnica de poste principal y compensación con fibra de los espacios intra-conducto. ${ }^{9}$

Entre las alternativas de individualización del poste, esta técnica semidirecta descripta permite reducir los riesgos de la retención del material de individualización dentro de alguna irregularidad del conducto que no fuera identificada y sería necesario el retiro de la restauración por desgaste con los consiguientes daños posibles y tiempo empleado.

Para minimizar estos inconvenientes el planteo de trabajar con una técnica de impresión semejante a una toma de patrón con método indirecto que los clínicos en general están habituados a utilizar y el vaciado inmediato con el material resiliente permiten rehabilitar en una sesión sin la necesidad de enviar al laboratorio y con la confección de un sistema de poste biomecánicamente próximo en compatibilidad a los tejidos dentarios. ${ }^{10}$

El tejido dentinario del conducto y remanente coronario tiene características complejas para lograr adhesión convencional.

La posible contaminación del procedimiento adhesivo durante la fase de acondicionamiento ácido y aplicación del sistema de adhesión para un cemento de resina dual pueden reducir la capacidad de unión.

Por estas razones el cementado del poste y núcleo coronario de resina se realizó con cemento autoadhesivo logrando un cementado con técnica sencilla y predecible en esta instancia donde la adaptación del poste es obtenida.

Esta solución además es compatible como sustrato estético para la restauración coronaria total seleccionada en material de cerámica sin metal resuelta con disilicato de litio.

El empleo de un poste colado metálico hubiera comprometido el resultado estético o limita la utilización de este tipo de restauración coronaria sin metal.

\section{Conclusión:}

La presentación de esta técnica semidirecta con materiales de acceso y manejo simple para todos los clínicos puede facilitar la resolución de algunas situaciones clínicas donde probablemente se encontraba limitada la aplicación de un sistema de poste de fibra y núcleo de resina.

Esta técnica permite una alternativa al tradicional poste metálico colado para solucionar a partir de materiales con mejor compatibilidad biomecánica, biológica y estética situaciones clínicas semejante a la expuesta.

\section{Bibliografía:}

1. Baba NZ, Goodacre CJ, Daher T. Restoration of endodontically treated teeth: the seven keys to success. Gen Dent 2009; 57:596-603.

2. Soares CJ, Valdivia AD, da Silva GR, et al. Longitudinal clinical evaluation of post systems: a literature review. Braz Dent J 2012;23:135-740.

3. R. Sarkis-Onofre, R. D. C. Jacinto, N. Boscato, M. S. Cenci, andT. Pereira-Cenci. Castmetal vs. glass fibre posts: a randomizedcontrolled trial with up to 3 years 
of follow up. Journal of Dentistry, 2014; 42, (5) 582-587.

4. Terry D, Triolo PT, Swift EJ. Fabrication of Direct Fiber-ReinforcedPosts: a structural design concept. J Esthet Restor Dent 2001; 13:228-240.

5. G. M. Gomes, R. V. Monte-Alto, G. O. Santos et al. Use of adirect anatomic post in a flared root canal: a three-year followup. Operative Dentistry, 2016 ;41 (1):23-28.

6. Boberick KG, Bachstein TK. Use of a flexible cast for the indirect fabrication of provisional restorations. J Prosthet Dent 1999; 82:90-3.

7. Chairside technique for expediting indirect interim restorations Dimashkieh, $\mathrm{M} R$, Rayyan MR The Journal of Prosthetic dentistry April 2016; 510-511.

8. Zanatta, RF., Tavares, RDP, Barreto, BC, Soares, C.J. Polymerization effect through translucent fiberglass posts in self-adhesive resin cements. J Dent Res. 2012, Vol 91 (Spec. Iss. B) Abstract \#2744.

9. D Torassa. Técnica de restauración con endoposte central y compensación de fibra. Rev Vision Estetica 2014; (4):28-37.

10. Arksornnukit, M., Jiangkongkho, P., Chaijareenont, P. Srisunakrua, L., Takahashi, H. Modulus of post affects fracture resistance of endodontically treated tooth. J Dent Res. 2010, Vol 89 (Spec. Iss. B) bstract \#2189.

\section{Palabras claves:}

ENDOPOSTES, CERÁMICA DENTAL, PRÓTESIS FIJA

\section{Keywords:}

ENDOPOSTS, DENTAL CERAMICS, FIXED PROTHETICS.

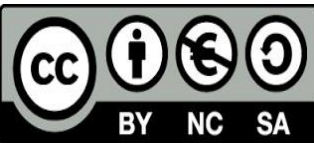

